\title{
Involvement of Apoptosis Inhibitor of Macrophages in a Rat Hypertension Model with Nephrosclerosis: Possible Mechanisms of Action of Olmesartan and Azelnidipine
}

\author{
Tadashi Uramatsu, ${ }^{a, b}$ Tomoya Nishino, ${ }^{* a}$ Yoko Obata,${ }^{a, c}$ Yohei Sato, ${ }^{d}$ Akira Furusu, ${ }^{a}$ \\ Takehiko Koji, ${ }^{e}$ Toru Miyazaki, ${ }^{d}$ and Shigeru Kohno ${ }^{a}$ \\ ${ }^{a}$ Second Department of Internal Medicine, Nagasaki University School of Medicine; ${ }^{b}$ Division of Blood Purification, \\ Nagasaki University Hospital; ${ }^{c}$ Medical Education Development Center, Nagasaki University Hospital; \\ Nagasaki 852-8501, Japan: ${ }^{d}$ Laboratory of Molecular Biomedicine for Pathogenesis, Center for Disease Biology and \\ Integrative Medicine, Faculty of Medicine, The University of Tokyo; Tokyo 113-0033, Japan: and ${ }^{e}$ Department of \\ Histology and Cell Biology, Nagasaki University Graduate School of Biomedical Sciences; Nagasaki 852-8523, \\ Japan. \\ Received November 2, 2012; accepted May 20, 2013
}

Stroke-prone spontaneously hypertensive (SHRsp) rats develop severe hypertension resulting in renal injury. We investigated apoptosis inhibitor of macrophages (AIM) expression in nephrosclerotic rats and the involvement of AIM in olmesartan (OLM)- and azelnidipine (AZN)-induced decreases in the number of macrophages infiltrating the kidney. We randomly assigned 20 -week-old male SHRsp rats to receive one of the following substances every day for 12 weeks: water (vehicle), hydralazine (HYD), OLM, or AZN. Renal damage was assessed by Masson trichrome staining. Expressions of ED-1, AIM, and oxidized low-density lipoprotein (oxLDL) were immunohistochemically detected. Apoptosis was analyzed by terminal deoxynucleotidyl transferase deoxyuridine triphosphate (dUTP) nick-end labeling (TUNEL) staining. All treatment groups showed significantly less renal interstitial fibrosis than the vehicle group. AZN and OLM groups had significantly fewer AIM-expressing cells than the HYD and vehicle groups. The ratios AIM-positive cells/ ED-1-positive macrophages and TUNEL-positive cells/ED-1-positive macrophages in the AZN and OLM groups were lower and higher, respectively, than the the HYD and vehicle groups. oxLDL expression in the renal interstitium was significantly lower in treatment groups compared to vehicle group. OLM and AZN inhibited interstitial fibrosis progression in SHRsp rats by suppressing AIM expression in macrophages, followed by reducing the number of infiltrating macrophages.

Key words apoptosis; macrophage; nephrosclerosis; oxidized low-density lipoprotein

The number of patients with chronic kidney disease (CKD) has increased dramatically worldwide. ${ }^{1)}$ A follow-up of the National Health and Nutrition Examination Surveys in the United States reported that the frequency of CKD (stages 1-4) increased from $10.0 \%$ in $1988-1994$ to $13.1 \%$ in $1999-2004 .^{2)}$ CKD is an independent risk factor (heart- and kidney-related) of cardiovascular disease, and is known to increase the risks for angina pectoris, myocardial infarction, cardiac arrest, cerebrovascular disorders, peripheral vascular disease, death due to cardiovascular disease, and sudden death. ${ }^{3,4)}$

Nephrosclerosis is one of the main diseases underlying CKD. ${ }^{5)}$ It involves progressive arteriosclerosis at the level of the small arteries and arterioles in the kidneys, leading to ischemic changes in the glomeruli and interstitium, consequently compromising renal function. ${ }^{6}$ This increased arteriosclerosis due to long-term hypertension associated with aging has resulted in the increased global incidence of nephrosclerosis.

Previous studies have reported that macrophages play a major role in the progression of arteriosclerosis in humans and in animal models. Further, macrophage infiltration is closely associated with the onset and progression of myocardial infarction, cerebrovascular disorders, and arteriosclerotic diseases such as nephrosclerosis. ${ }^{7-12)}$

Apoptosis inhibitor of macrophages (AIM) is a secretory protein comprising 3 scavenger receptor cystein-rich domains.

The authors declare no conflict of interest.
It is specifically produced by mature tissue macrophages, ${ }^{13)}$ and supports the survival of macrophages themselves against different apoptosis-inducing stimuli. AIM has recently attracted attention as a new protein involved in macrophage apoptosis. Incorporation of oxidized low-density lipoprotein (oxLDL) increases the expression of AIM in macrophages. ${ }^{14)}$ Recently, AIM was found to be strongly expressed in macrophages that infiltrated atherosclerotic lesions in humans and animal models, and this inhibitor may be intimately involved in atherosclerosis. ${ }^{14)}$

Recent studies have revealed that in addition to the antihypertensive effects, renin-angiotensin inhibitors and some calcium antagonists exert cardiovascular or renoprotective actions in patients with arteriosclerosis. ${ }^{15,16)}$ Among the angiotensin II receptor blockers, olmesartan (OLM) is reported to have high affinity to the angiotensin II type 1 receptor. Meanwhile, azelnidipine (AZN) is a newly developed long-acting dihydropyridine-type calcium channel blocker (CCB) with a hypotensive effect that is comparable to that of other common CCBs. These drugs have shown anti-oxidant or anti-inflammatory effects that are independent of the anti-hypertensive effect. ${ }^{17,18)}$ Thus, we previously investigated the renoprotective effect of OLM and AZN in nephrosclerotic rats. We found significant decreases in the number of infiltrating macrophages and inhibition of interstitial fibrosis in groups of the same model treated with OLM or AZN compared to the rats treated with hydralazine (HYD), which has low anti-oxidant and anti-inflammatory effects. This effect was not associated 
with the extent of proteinuria or antihypertensive effect. ${ }^{19)}$ Hence, it was suggested that OLM and AZN protect the kidneys by a mechanism other than hypotensive activity alone. However, it remains to be elucidated whether or not AIM is involved in reducing the number of infiltrating macrophages in the renal tissues.

Here, we evaluated the expression of AIM in a rat model of nephrosclerosis and investigated its possible involvement in OLM- and AZN-induced decreases in the number of macrophages infiltrating the renal interstitium.

\section{MATERIALS AND METHODS}

Animals In this study, we used 20-week-old male strokeprone spontaneously hypertensive (SHRsp)/Izm rats (Japan SLC, Shizuoka, Japan) and normotensive control WistarKyoto (WKY) rats. SHRsp was first isolated from WKY rats by Okamoto and Aoki in 1963. ${ }^{20)}$ All the rats were housed in standard rodent cages at a constant ambient temperature $\left(22 \pm 1^{\circ} \mathrm{C}\right)$ and humidity $(85 \%)$, with 12 -h cycles of light and dark and ad libitum access to Funabashi SP diet (Funabashi Farm, Chiba, Japan) and tap water. The experimental protocol was reviewed and approved by the Animal Care and Use Committee of Nagasaki University School of Medicine (No. 1004050846). Further, the study protocol complied with the laws and notifications of the Japanese government prior to commencement of the study.

Drugs AZN and OLM were donated by Sankyo Co., Ltd. (Tokyo, Japan).

Experimental Protocol Rats were treated with $10 \mathrm{mg} /$ $(\mathrm{kg} \cdot \text { day })^{-1}$ AZN $(n=6), 3 \mathrm{mg} /(\mathrm{kg} \cdot \text { day })^{-1}$ OLM $(n=4), 20 \mathrm{mg} /$ $(\mathrm{kg} \cdot \text { day })^{-1}$ hydralazine (HYD) (Sigma, St. Louis, MO, U.S.A.) $(n=3)$, or water (vehicle; $n=5)$. AZN and OLM were suspended in $0.5 \%$ carboxymethylcellulose (039-01335; Wako Pure Chemical Industries, Ltd., Osaka, Japan). Previous reports have indicated that treatment with carboxymethylcellulose does not affect blood pressure, and has no apparent inhibitory effects on urinary protein excretion or renal injury in SHRsp. ${ }^{21)}$ Each agent was administered by gavage for 12 weeks. At 32 weeks of age, the rats were sacrificed and their kidneys were collected. In this study, the dose of each drug and the time schedule was decided based on our previous study. $^{19)}$

Histological Examination Some sections of the kidney tissue were fixed in 4\% paraformaldehyde (PFA) in phosphatebuffered saline (PBS) ( $\mathrm{pH}$ 7.4). The other sections were frozen in OCT compound (Miles, Elkhart, IN, U.S.A.) and stored at $-80^{\circ} \mathrm{C}$. The fixed renal tissues were embedded in paraffin and cut into $4-\mu \mathrm{m}$-thick sections. The sections were then stained with Masson trichrome stain to reveal histological changes and areas of interstitial fibrosis.

Terminal Deoxyribonucleotidyl Transferase (TdT)Mediated Biotin-16-deoxyuridine Triphosphate (dUTP) Nick-End Labeling (TUNEL) Assay Apoptosis was determined on $4-\mu \mathrm{m}$-thick paraffin-embedded kidney sections using TUNEL in an in situ apoptosis detection kit (TaKaRa Bio, Otsu, Shiga), as reported previously. ${ }^{22,23)}$ In each group, the number of TUNEL-positive cells was counted in 20 nonoverlapping fields under $\times 200$ magnification.

Antibodies The immunohistochemical procedure used in this study was described previously. ${ }^{24,25)}$ The following anti- bodies were used for immunohistochemistry: mouse anti-ED-1 antibody (1:100 dilution, MCA341R; Serotec, Oxford, U.K.) as a marker of rat macrophages, rabbit anti-AIM polyclonal antibody (1:500 dilution, SA-2, donated by Prof. T. Miyazaki), rabbit anti-oxLDL polyclonal antibody (1:200 dilution, AB3230; Chemicon International, Temecula, CA, U.S.A.) as a marker of oxidant injury, and goat anti-macrophage chemotactic protein (MCP)-1 polyclonal antibody (1:20 dilution, sc1784; Santa Cruz Biotechnology, Inc., Santa Cruz, CA, U.S.A.).

Immunohistochemical Study For immunohistochemistory, 4- $\mu \mathrm{m}$-thick paraffin-embedded tissues were deparaffinized and blocked for endogenous peroxidases by immersing the slides in $0.3 \%$ hydrogen peroxide $\left(\mathrm{H}_{2} \mathrm{O}_{2}\right)$ in methanol for 20 min. Fresh-frozen sections fixed in $4 \%$ PFA were also blocked for endogenous peroxidases by the same method.

The tissue sections were immunohistochemically stained using an indirect method to assess ED-1, AIM, and MCP-1. To reduce nonspecific background staining, the sections were incubated with a blocking buffer containing $10 \%$ normal goat serum (X0907; DakoCytomation Denmark A/S, Glostrup, Denmark), 10\% fetal calf serum (011-16290M; Gibco Life Technology, Carlsbad, CA, U.S.A.), and 2\% normal rat serum (X0912; Dako) in PBS for $30 \mathrm{~min}$. Sections were then treated with the primary antibody, which was diluted in the same blocking buffer. After reacting with anti-ED-1 or anti-AIM antibodies at room temperature for $1 \mathrm{~h}$, the sections were treated with horseradish peroxidase (HRP)-conjugated rabbit anti-mouse antibodies (1:100 dilution, P0161; Dako) and HRP-conjugated swine anti-rabbit antibodies (P0399; Dako) at room temperature for $30 \mathrm{~min}$. After reaction with anti-MCP-1 antibodies at room temperature for $1 \mathrm{~h}$, the sections were treated with HRP-conjugated rabbit anti-goat antibodies (1:100 dilution, P0449; Dako) at room temperature for $1 \mathrm{~h}$.

The peroxidase/anti-peroxidase method was used to assess the oxLDL expression. For this analysis, fresh-frozen sections were fixed in $4 \%$ PFA in PBS ( $\mathrm{pH} 7.4$ ) at room temperature for $15 \mathrm{~min}$ and then incubated with blocking buffer for 30 min, similar to the process described above. After reacting with anti-oxLDL antibodies at room temperature for $1 \mathrm{~h}$, signal detection was carried out using the EnVision Plus System (K4008; Dako).

$\mathrm{H}_{2} \mathrm{O}_{2}$ and 3,3'-diaminobenzidine tetrahydrochloride (DAB) were used as the chromogen for ED-1, AIM, and oxLDL. HRP-positive sites in MCP-1 were visualized using $\mathrm{H}_{2} \mathrm{O}_{2}$ and $\mathrm{DAB}$ in the presence of nickel and cobalt ions. Finally, the sections were counterstained with methyl green and mounted.

Negative control sections were reacted with normal mouse immunoglobulin $\mathrm{G}$ ( $\mathrm{IgG}$ ) instead of specific monoclonal antibodies or with normal rabbit or goat IgG in place of specific polyclonal antibodies.

To identify AIM-positive cells among the ED-1-positive cells in the tubulointerstitium, double immunolabeling for AIM and ED-1 was performed using the methods described by Furusu et al., ${ }^{26)}$ with a slight modification. Briefly, deparaffinized peritoneal sections were incubated with blocking serum and anti-ED-1 antibody. The sections were then washed with PBS and incubated with the biotinylated antimouse antibody. After washing, the sections were incubated with alkaline phosphatase-conjugated avidin (Vector Laboratories, Burlingame, CA, U.S.A.), following a reaction with $\mathrm{BCIP} /$ 

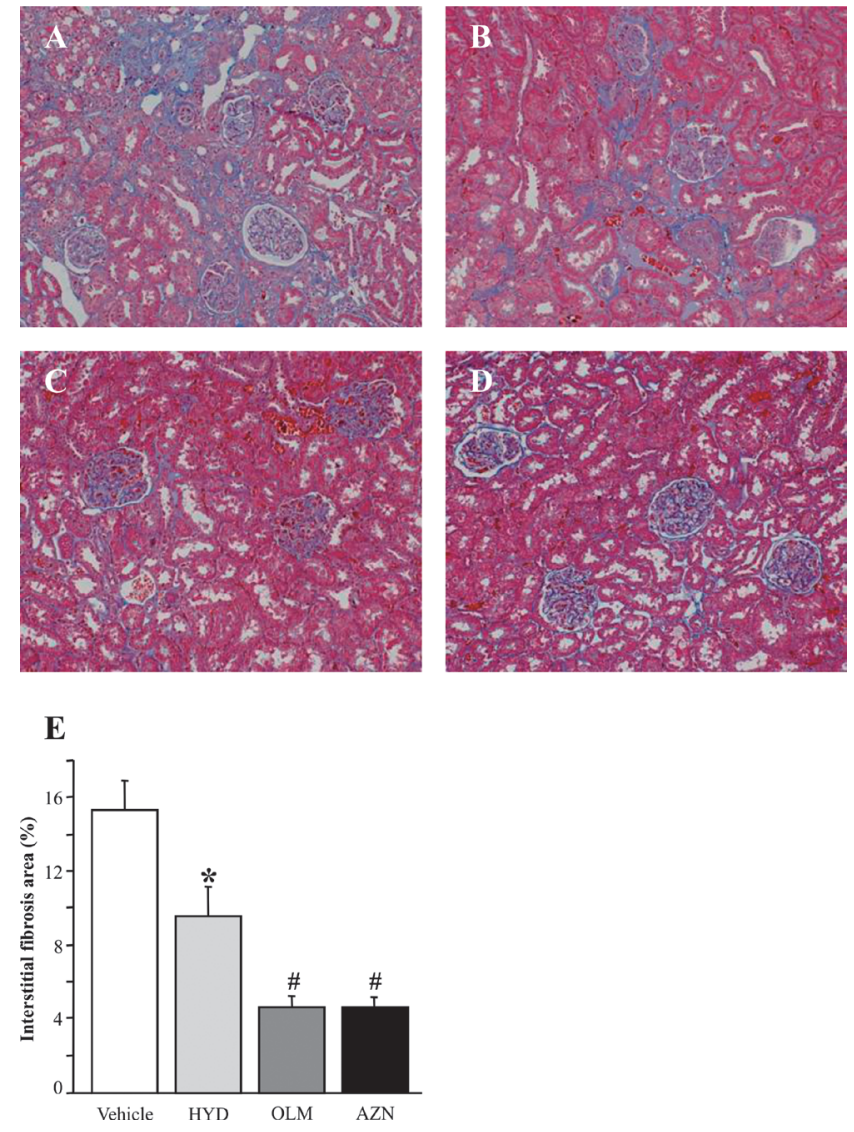

Fig. 1. Masson Trichrome Staining

Treatment with antihypertensive drugs significantly decreased the fibrotic area in SHRsp rats. The interstitial fibrotic areas in the OLM (C) and AZN (D) groups were significantly lower than those in the vehicle SHRsp (A) and HYD (B) groups, as indicated by the quantitative analysis of the interstitial fibrotic areas in the kidneys among the four groups (E). Data represent mean \pm S.E.M. ${ }^{*} p<0.05 v s$. vehicle; $\# p<0.05$ vs. vehicle and HYD groups.

NBT (Dako). After staining for ED-1, tissue sections were stained for AIM using the avidin-biotin complex kit, as described earlier.

Image Analysis For semiquantitative estimation of the blue area of Masson trichrome staining and the area of positive expression for each protein, images were digitized using image analysis software (Win Roof Mitanicorp, Chiba, Japan). The images were transformed into a $2250 \times 1800$ pixel matrix and viewed at $\times 100$ magnification; the area of each section was $0.89 \mathrm{~mm}^{2}$. The area of cortical interstitium was measured by light microscopic examination. For each group, 20 such areas were selected. Of a total of 80 fields, we calculated the percentage of areas that stained positive for oxLDL and MCP-1, and interstitial fibrosis by Masson trichrome stain. We also counted the number of ED-1-positive macrophages and AIM-expressing macrophages at 20 fields under $\times 200$ magnification in each group.

Statistical Analysis Data are expressed as the mean \pm standard error of the mean (S.E.M.). Differences among groups were examined for statistical significance using repeated-measures analysis of variance (ANOVA) with Fisher's protected least significant difference. Differences were considered statistically significant at $p<0.05$.
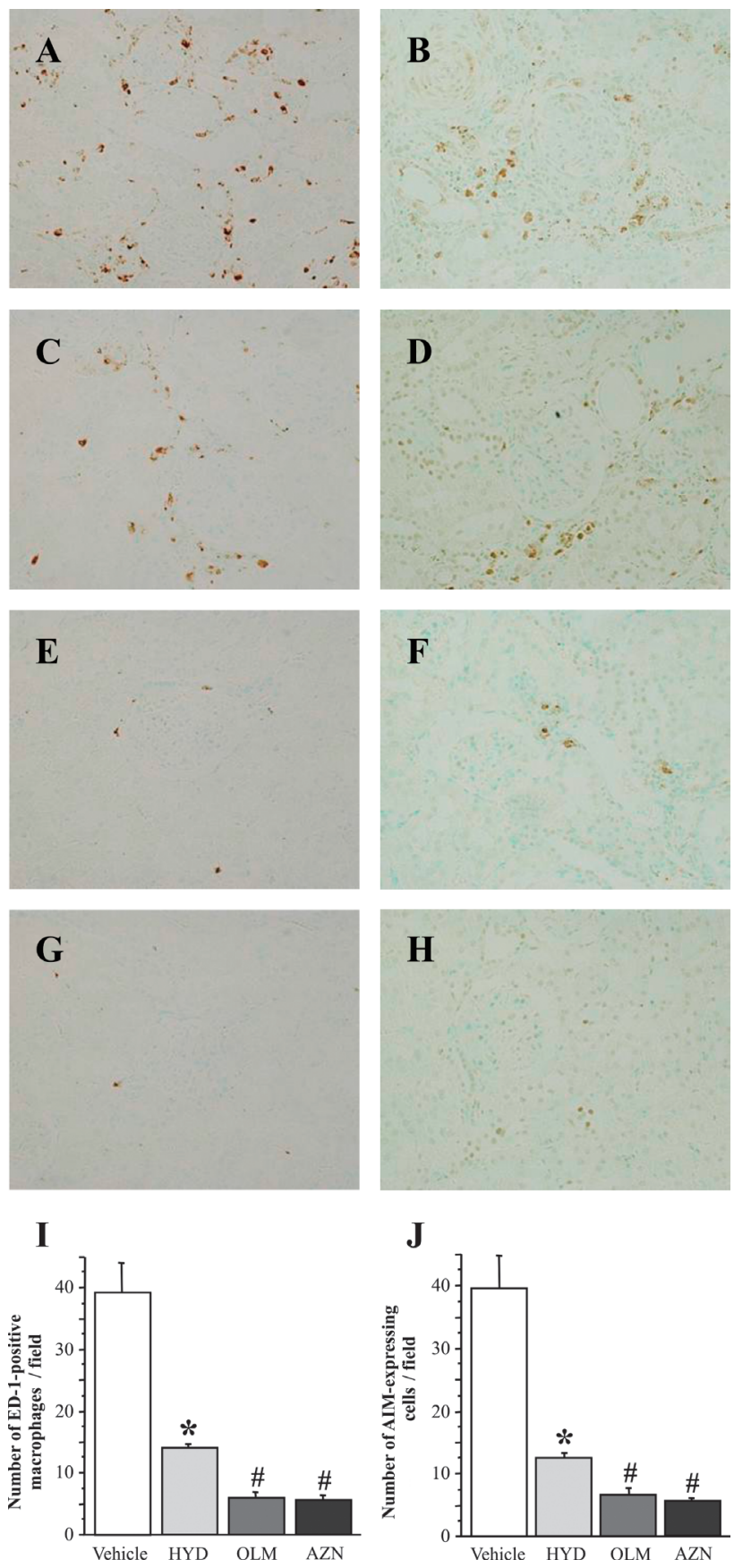

Fig. 2. ED-1 and AIM (Apoptosis Inhibitor of Macrophages) Staining

ED-1 staining revealed macrophage infiltration in the tubulointerstitium of the vehicle SHRsp rats (A), but this was markedly lower in rats treated with HYD (C), OLM (E), or AZN (G). Macrophage infiltration in the OLM (E) and AZN (G) groups was significantly lower than that in the HYD (C) group. Immunohistochemical staining showed that the OLM (F) and AZN (H) groups had significantly fewer AIM-expressing cells than the HYD (D) and vehicle (B) groups. (I) and (J) present the quantitative analyses of the number of ED-1-positive macrophages and AIMexpressing cells, respectively, in the kidneys among the four groups. Data represent mean \pm S.E.M. ${ }^{*} p<0.05$ vs. vehicle; \# $p<0.05$ vs. vehicle and HYD groups.

\section{RESULTS}

Morphological Examinations In SHRsp rats, the degree of interstitial fibrosis increased from 16-30 weeks of age, which was different from that observed in WKY rats (data not shown). Masson trichrome staining revealed marked matrix expansion in glomeruli and interstitial fibrosis in the vehicle group (Fig. 1A). Interstitial fibrosis was locally inhibited in 
A

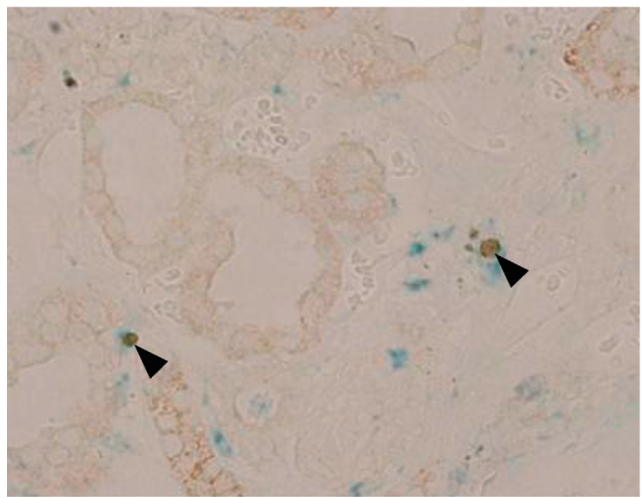

B

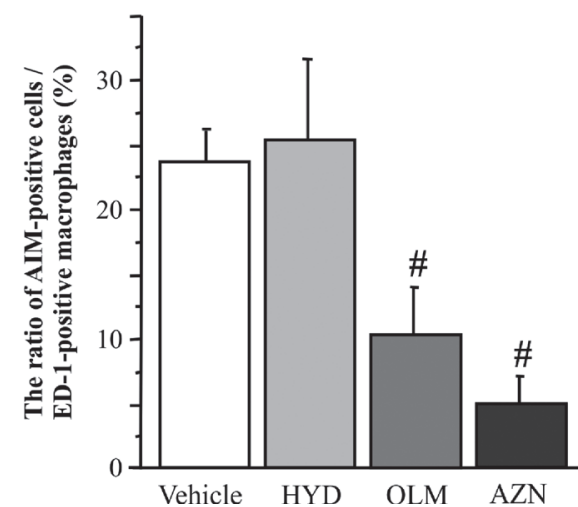

Fig. 3. Double Staining for AIM (Brown) and ED-1 (Blue)

AIM-positive cells were detected among ED-1-positive cells by immunostaining for both AIM and ED-1. AIM-positive cells stained brown, while ED-1-positive cells stained blue (A). The black arrowheads indicate AIM- and ED-1-positive macrophages. The ratios of AIM-positive cells to ED-1-positive macrophages in the OLM and AZN groups were lower than those in the HYD and vehicle groups (B). Data represent means \pm S.E.M. \#p<0.05 vs. vehicle and HYD groups.
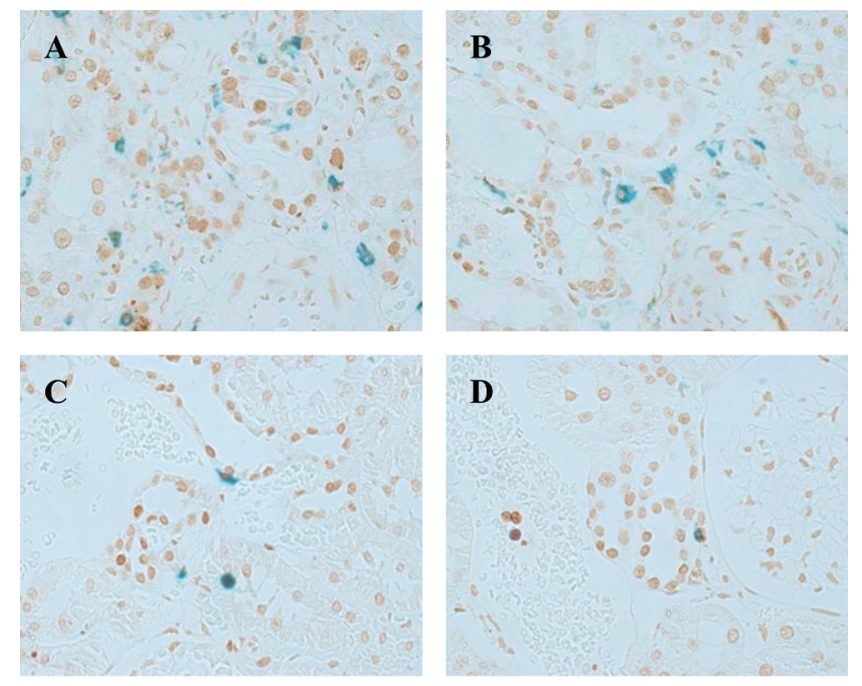

$\mathbf{E}$

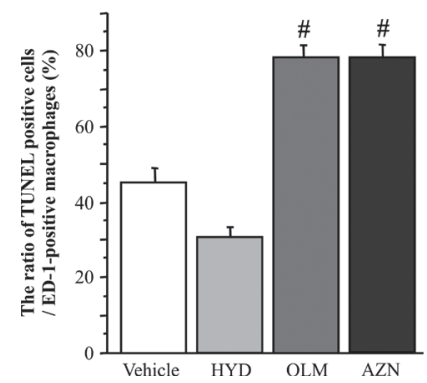

Fig. 4. Double Staining for TUNEL (Brown) and ED-1 (Blue)

To detect TUNEL-positive cells among ED-1-positive cells, double immunostaining for TUNEL and ED-1 was performed (A-D). The ratio of TUNEL-positive cells to ED-1-positive macrophages (E) was higher in the OLM (C) and AZN (D) groups than in the HYD (B) and vehicle (A) groups, as indicated by the quantitative analysis of the ratio of TUNEL-positive cells to ED-1-positive macrophages in the kidneys among the four groups (E). Data represent mean \pm S.E.M. $\# p<0.05$ vs. vehicle and HYD groups.

the HYD group (Fig. 1B) and dramatically inhibited in the OLM (Fig. 1C) and AZN (Fig. 1D) groups. Semiquantitative analysis revealed that the HYD, AZN, and OLM groups showed significantly decreased area of interstitial fibrosis as compared with the vehicle. Moreover, interstitial fibrosis was
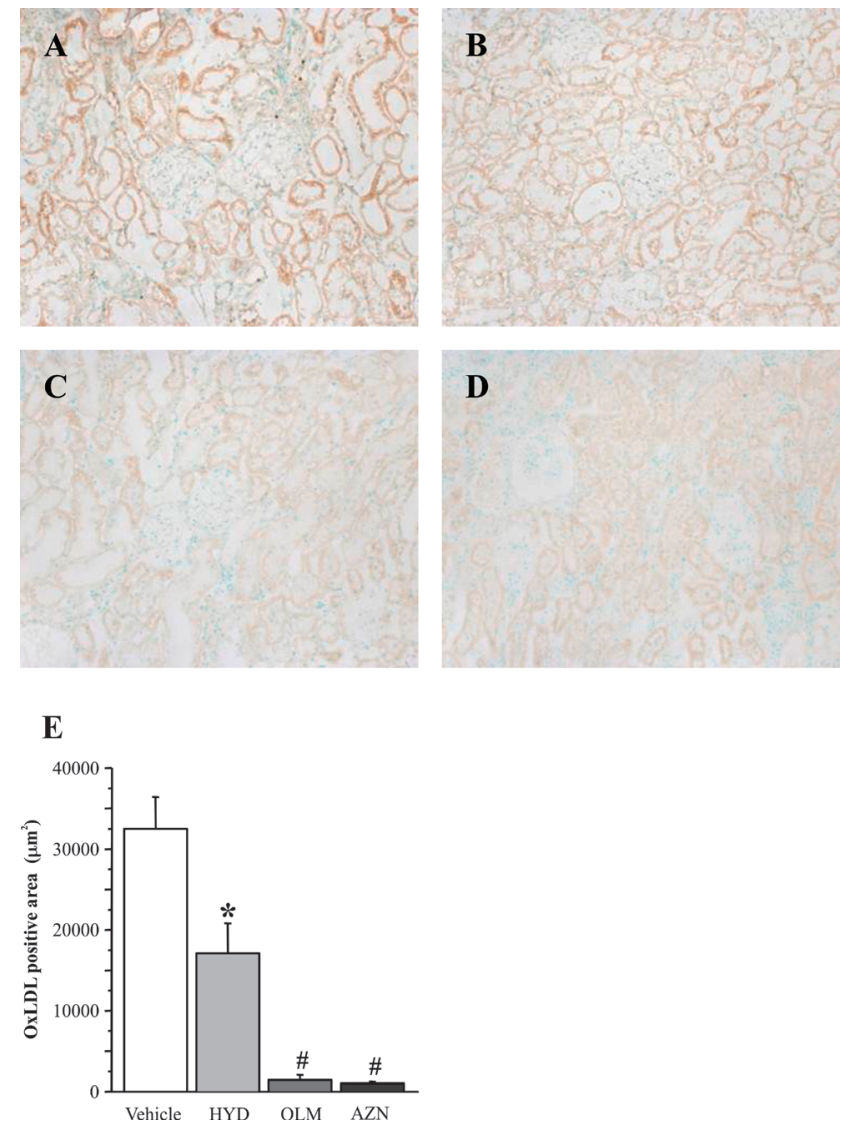

Fig. 5. oxLDL Expression

Markedly increased oxLDL expression was observed in the tubular cells in the vehicle group (A). Treatment with antihypertensive drugs significantly decreased the oxLDL expression in SHRsp rats. oxLDL expression was significantly lower in the OLM (C) and AZN (D) groups than in the HYD (B) group, as indicated by the quantitative analysis of oxLDL positive area in the kidneys among the four groups (E). Data represent mean \pm S.E.M. ${ }^{*} p<0.05$ vs. vehicle; $\# p<0.05$ vs. vehicle and HYD groups.

significantly suppressed in the AZN and OLM groups as compared to the HYD group (Fig. 1E).

Macrophage Infiltration In a previous study, development of hypertension and interstitial fibrosis was found to be associated with macrophage infiltration in the kidney of 

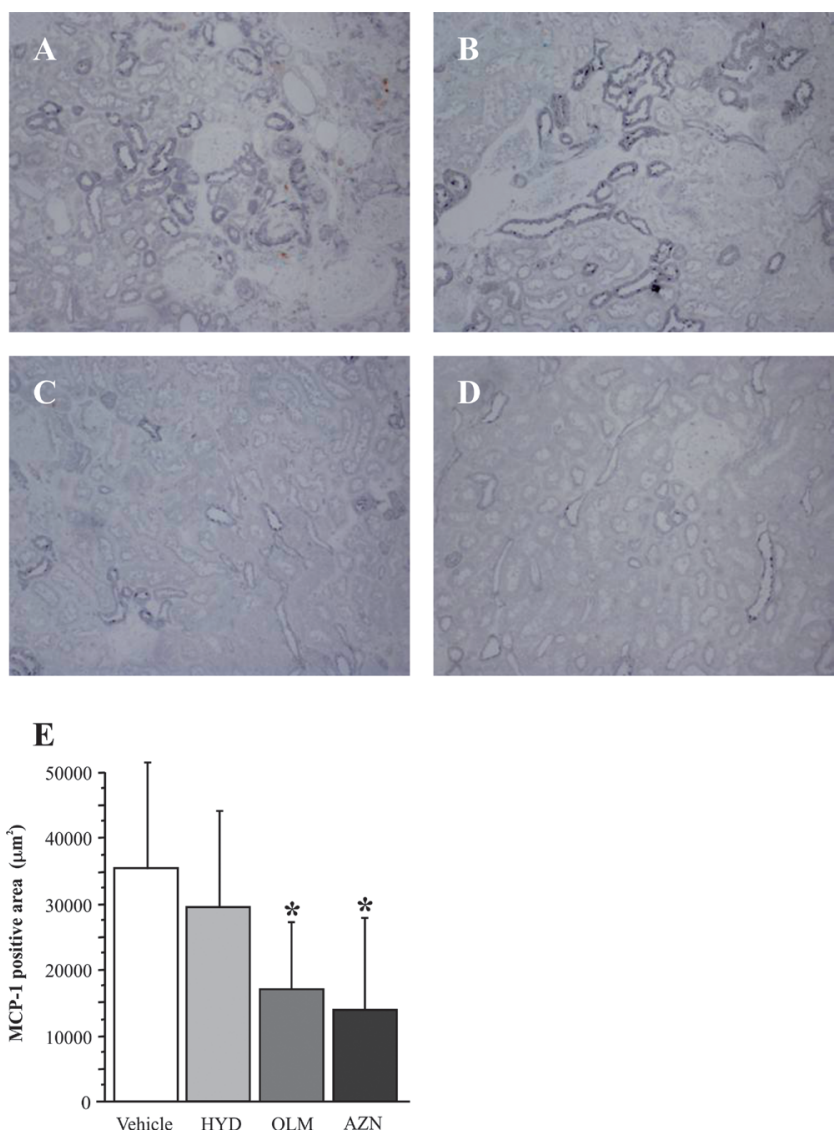

Fig. 6. MCP-1 Expression

Increased MCP-1 expression was observed in the tubular cells in the vehicle group (A). MCP-1 expression was not significantly different between the HYD (B) and vehicle (A) groups. MCP-1 expression was significantly lower in the OLM (C) and AZN (D) groups than in the vehicle group (A), as indicated by the quantitative analysis of MCP-1 positive area in the kidneys among the four groups (E). Data represent mean \pm S.E.M. $* p<0.05 v s$. vehicle.

SHRsp rats. ${ }^{19)}$ We examined the effects of antihypertensive agents on macrophage infiltration by immunohistochemistry for the macrophage marker ED-1. Many macrophages were present in the tubulointerstitium of the vehicle group (Fig. 2A), but the number of ED-1-positive macrophages was markedly decreased in rats treated with antihypertensive agents as compared to that in the vehicle group. Furthermore, macrophage infiltration was significantly reduced in the OLM (Fig. 2E) and AZN (Fig. 2G) groups as compared to the HYD group (Fig. 2C).

Involvement of AIM in Macrophage Infiltration To clarify the mechanisms underlying suppression of macrophage infiltration observed in rats treated with antihypertensive agents, we performed immunohistochemical analysis for AIM expression. WKY rats showed few AIM-expressing cells (data not shown). In addition, we observed that AIM-positive cells predominantly infiltrated in the interstitium and there were few AIM-positive cells in the glomerulus (data not shown). The number of AIM-expressing cells in the vehicle group was markedly higher than that in WKY rats (Fig. 2B). Conversely, the number of AIM-expressing cells in the OLM (Fig. 2F) and AZN (Fig. 2H) groups was significantly lower than that in the HYD (Fig. 2D) and vehicle groups.

We performed double-staining for AIM and ED-1 (Fig. 3A) to ascertain the percentage of AIM-expressing cells among macrophages. The results revealed that the ratio of AIMpositive cells to ED-1-positive macrophages was significantly lower in the OLM and AZN groups than in the HYD and vehicle groups (Fig. 3B).

The number of apoptotic macrophages was then investigated, as AIM-positive cells were markedly decreased in the OLM and AZN groups. Double immunostaining for TUNEL and ED-1 was performed in the same section (Figs. 4A-D). The ratio of TUNEL-positive cells to ED-1-positive macrophages was increased in the OLM (Fig. 4C) and AZN (Fig. 4D) groups in comparison to the HYD (Fig. 4B) and vehicle (Fig. 4A) groups.

Expression of oxLDL AIM expression has recently been reported to be regulated via liver $\mathrm{X}$ receptors (LXR)/retinoid $\mathrm{X}$ receptors (RXR) and incorporation of oxLDL yielding oxysterols, the native ligands of LXR, also upregulate AIM expression in macrophages. We investigated the expression of oxLDL, which affects AIM expression in antihypertensive agent-treated rats. In the vehicle group, strong expression of oxLDL was observed in tubular cells (Fig. 5A), whereas expression was significantly reduced in the HYD (Fig. 5B), OLM (Fig. 5C), and AZN (Fig. 5D) groups. Furthermore, expression of oxLDL was significantly lower in the OLM (Fig. 5C) and AZN (Fig. 5D) groups compared to that in the HYD (Fig. 5B) group.

Expression of MCP-1 In addition, we examined the MCP-1 expression, as a chemotactic factor of macrophages. In the vehicle group, increased expression of MCP-1 was observed in tubulointerstitial area (Fig. 6A). Furthermore, expression of MCP-1 was significantly lower in the OLM (Fig. 6C) and the AZN (Fig. 6D) groups compared to that in the vehicle group (Fig. 6A), whereas the expression was not significantly reduced in the HYD (Fig. 6B) compared to that in the vehicle group (Fig. 6A).

\section{DISCUSSION}

In this study, we found significantly fewer infiltrating macrophages and dramatic inhibition of interstitial fibrosis following treatment with OLM or AZN but not HYD. This finding strongly demonstrated that OLM and AZN have mechanisms distinct from the expected antiproteinuric or antihypertensive effect reported in our previous study. ${ }^{19)}$ Moreover, we showed that AIM expression in macrophages in the renal tissue of SHRsp rats seemed closely correlated with the number of infiltrating macrophages, and both these drugs significantly decreased the number of AIM-positive macrophages as well as the total number of macrophages, suggesting that AIM expression is vital to the progression of nephrosclerosis.

It is well known that glomerular or interstitial macrophage infiltration is a prominent feature in nephrosclerosis, diabetic nephropathy, and lupus nephritis. ${ }^{27)}$ However, the roles of macrophages in these renal diseases are quite controversial. A previous study has reported that infiltrating macrophages play a significant role in tissue repair or remodeling. ${ }^{28)}$ Meanwhile, macrophages have also been reported to produce cytokines, nitric oxide, and reactive oxygen species (ROS), ${ }^{29)}$ causing direct damage to resident interstitial fibroblasts and myofibroblasts (which are a primary source of extracellular matrix proteins), consequently facilitating the progression of renal disorders. ${ }^{9-12)}$ In SHRsp rats, we previously reported 
that the number of ED-1-positive macrophages was markedly increased in glomeruli and interstitial fibrosis areas. ${ }^{19)}$ Therefore, we considered that macrophages promote the progression of interstitial fibrosis in SHRsp rats.

Some studies have investigated the role of ROS in the progression of renal injury. A report on the effect of ROS derived from macrophages on renal disorders indicated that ROS cause proteinuria and damage consisting of necrosis of endothelial cells with platelet activation and proliferation of resident glomerular cells. In addition, ROS have been reported to be actively involved in the activation of chemotactic factors and adhesion molecules. ${ }^{30)}$ Our previous report also showed increased oxidative stress as well as involvement in interstitial fibrosis in the rat model of nephrosclerosis. ${ }^{19)}$ In the present study, we confirmed the increased expression of oxLDL, which is produced by ROS, in SHRsp rats.

Further, it is noteworthy that AIM was expressed in macrophages infiltrating renal tissue; therefore, the macrophages were supposed to exhibit resistance to apoptosis. ${ }^{14)}$ According to previous literature, oxLDL is widely known as an inducer of AIM expression. As ROS in blood increase due to oxidative stress, the oxLDL level continues to increase and it binds to LXR/RXR in nuclear receptors following uptake by macrophages to induce AIM gene expression and stimulate AIM production/secretion in macrophages. ${ }^{14)}$ In fact, our immunohistochemical results revealed strong oxLDL expression primarily in the renal tubules and increase in the number of AIM-expressing macrophages in the vehicle group. These findings may suggest that AIM plays an important role in controlling the number of infiltrating macrophages by inhibiting macrophage apoptosis in SHRsp rats. On the other hand, oxLDL expression was significantly lower and there were fewer macrophages in OLM- and AZN-treated rats as compared to the corresponding parameters in the vehicleand HYD-treated rats. One explanation for this result could be the antioxidant action of OLM and AZN, which occurs by inducing superoxide dismutase activity in blood vessels ${ }^{31}$ ) or by suppressing ROS production. ${ }^{32-41)}$ OLM is known to block angiotensin II induced superoxide production by reduced nicotinamide adenine dinucleotide phosphate (NADPH) oxidase. $^{42)}$ In addition, AZN has reported to attenuate ROS production via suppression of NADPH oxidase in a manner similar to OLM, ${ }^{19,32}$ and is a more potent antioxidant than any other dihydropyridine calcium antagonists because of its good liposolubility and vascular endothelial penetration. ${ }^{18)}$ Hence, it is speculated that OLM and AZN indirectly suppress AIM expression in macrophages via their inhibitory effects on oxLDL expression. The resultant increase in macrophage apoptosis then led to a decrease in the number of macrophages in renal tissue, thus inhibiting interstitial fibrosis. In addition, as another mechanism leading to macrophage infiltration in the kidney of SHRsp rats, increase in the MCP-1 expression has been reported to be caused by oxidant stress. ${ }^{9)}$ Similar to these results, we found increased MCP-1 expression in the vehicle group and decreased expression in the OLM and AZN groups. Based on these findings, we considered that the antioxidant effects of OLM and AZN suppressed not only AIM expression but also MCP-1 expression; further, these drugs reduced the number of infiltrating macrophages, resulting in the inhibition of interstitial fibrosis ${ }^{32-41)}$. Currently, it is not yet clarified which drug, OLM or AZN, is stronger on antioxidant effects, future studies comparing OLM and AZN are required to determine which of these is a stronger antioxidant.

Recently, it has been shown that combination therapy with OLM and AZN is more effective than monotherapy with either drug for reducing macrophage infiltration and interstitium disorders in renal failure models. ${ }^{29)}$ Moreover, this combination therapy was also reported to inhibit MCP-1 and tumor necrosis factor- $\alpha$ expressions more effectively in the mouse vascular injury model. ${ }^{18)}$ Thus, although this was not investigated in the current study, OLM and AZN combination therapy may lower oxidative stress and inhibit AIM expression to a greater extent than monotherapy, leading to further decrease in the number of macrophages and more effective inhibition of interstitial fibrosis. Further studies are warranted to investigate the effectiveness of such combination therapy.

In conclusion, our study demonstrated that OLM and AZN treatment can reduce the number of infiltrating macrophages and inhibit interstitial fibrosis. AIM is involved in the progression of interstitial fibrosis in SHRsp rats. The oxLDL-inhibiting action of these 2 drugs appears to result in the observed decrease in AIM expression.

Acknowledgment The authors wish to thank Ms. Tomomi Kurashige, Ms. Ryoko Yamamoto, and Ms. Shiho Kondo for their excellent technical assistance.

\section{REFERENCES}

1) Meguid El Nahas A, Bello AK. Chronic kidney disease: the global challenge. Lancet, 365, 331-340 (2005).

2) Coresh J, Selvin E, Stevens LA, Manzi J, Kusek JW, Eggers P, Van Lente F, Levey AS. Prevalence of chronic kidney disease in the United States. JAMA, 298, 2038-2047 (2007).

3) Go AS, Chertow GM, Fan D, McCulloch CE, Hsu CY. Chronic kidney disease and the risks of death, cardiovascular events, and hospitalization. N. Engl. J. Med., 351, 1296-1305 (2004).

4) Ninomiya T, Kiyohara Y, Kubo M, Tanizaki Y, Doi Y, Okubo K, Wakugawa Y, Hata J, Oishi Y, Shikata K, Yonemoto K, Hirakata H, Iida M. Chronic kidney disease and cardiovascular disease in a general Japanese population: the Hisayama Study. Kidney Int., 68, 228-236 (2005).

5) Nakaya H, Sasamura H, Hayashi M, Saruta T. Temporary treatment of prepubescent rats with angiotensin inhibitors suppresses the development of hypertensive nephrosclerosis. J. Am. Soc. Nephrol., 12, 659-666 (2001).

6) Mimura I, Nangaku M. The suffocating kidney: tubulointerstitial hypoxia in end-stage renal disease. Nat. Rev. Nephrol., 6, 667-678 (2010).

7) Moreno PR, Falk E, Palacios IF, Newell JB, Fuster V, Fallon JT. Macrophage infiltration in acute coronary syndromes. Implications for plaque rupture. Circulation, 90, 775-778 (1994).

8) Stoll G, Bendszus M. Inflammation and atherosclerosis: novel insights into plaque formation and destabilization. Stroke, 37, 19231932 (2006).

9) Hilgers KF, Hartner A, Porst M, Mai M, Wittmann M, Hugo C, Ganten D, Geiger H, Veelken R, Mann JF. Monocyte chemoattractant protein-1 and macrophage infiltration in hypertensive kidney injury. Kidney Int., 58, 2408-2419 (2000).

10) Hartner A, Porst M, Klanke B, Cordasic N, Veelken R, Hilgers KF. Angiotensin II formation in the kidney and nephrosclerosis in Ren-2 hypertensive rats. Nephrol. Dial. Transplant., 21, 1778-1785 (2006).

11) Rodriguez-Iturbe B, Johnson RJ. Role of inflammatory cells in the kidney in the induction and maintenance of hypertension. Nephrol. Dial. Transplant., 21, 260-263 (2006). 
12) Imakiire $T$, Kikuchi $Y$, Yamada $M$, Kushiyama $T$, Higashi $K$, Hyodo N, Yamamoto K, Oda T, Suzuki S, Miura S. Effects of renin-angiotensin system blockade on macrophage infiltration in patients with hypertensive nephrosclerosis. Hypertens. Res., 30, 635-642 (2007).

13) Miyazaki T, Hirokami $Y$, Matsuhashi N, Takatsuka H, Naito M. Increased susceptibility of thymocytes to apoptosis in mice lacking AIM, a novel murine macrophage-derived soluble factor belonging to the scavenger receptor cysteine-rich domain superfamily. J. Exp. Med., 189, 413-422 (1999).

14) Arai S, Shelton JM, Chen M, Bradley MN, Castrillo A, Bookout AL, Mak PA, Edwards PA, Mangelsdorf DJ, Tontonoz P, Miyazaki T. A role for the apoptosis inhibitory factor AIM/Spalpha/Api6 in atherosclerosis development. Cell Metab., 1, 201-213 (2005).

15) Dahlöf B, Devereux RB, Kjeldsen SE, Julius S, Beevers G, de Faire U, Fyhrquist F, Ibsen H, Kristiansson K, Lederballe-Pedersen O, Lindholm LH, Nieminen MS, Omvik P, Oparil S, Wedel H, LIFE Study Group. Cardiovascular morbidity and mortality in the Losartan Intervention For Endpoint reduction in hypertension study (LIFE): a randomised trial against atenolol. Lancet, 359, 995-1003 (2002).

16) Casas JP, Chua W, Loukogeorgakis S, Vallance P, Smeeth L, Hingorani AD, MacAllister RJ. Effect of inhibitors of the renin-angiotensin system and other antihypertensive drugs on renal outcomes: systematic review and meta-analysis. Lancet, 366, 2026-2033 (2005).

17) Jinno T, Iwai M, Li Z, Li JM, Liu HW, Cui TX, Rakugi H, Ogihara T, Horiuchi M. Calcium channel blocker azelnidipine enhances vascular protective effects of AT1 receptor blocker olmesartan. Hypertension, 43, 263-269 (2004).

18) Inaba S, Iwai M, Tomono Y, Senba I, Furuno M, Kanno H, Okayama H, Mogi M, Higaki J, Horiuchi M. Prevention of vascular injury by combination of an AT1 receptor blocker, olmesartan, with various calcium antagonists. Am. J. Hypertens., 22, 145-150 (2009).

19) Kurashige T, Abe K, Furusu A, Miyazaki M, Obata Y, Xia Z, Nakazawa M, Nakazawa Y, Funakoshi S, Harada T, Koji T, Kohno S. Renoprotective effect of azelnidipine in rats. Biol. Pharm. Bull., 31, 2237-2244 (2008).

20) Okamoto K, Aoki K. Development of a strain of spontaneously hypertensive rats. Jpn. Circ. J., 27, 282-293 (1963).

21) Oizumi K, Miyamoto $\mathrm{M}$, Koike $\mathrm{H}$. Effects of dihydropyridine $\mathrm{Ca}$ blockers on the renal function in nephrotic spontaneously hypertensive rat (SHR). Biol. Pharm. Bull., 17, 407-410 (1994).

22) An S, Hishikawa Y, Liu J, Koji T. Lung injury after ischemiareperfusion of small intestine in rats involves apoptosis of type II alveolar epithelial cells mediated by TNF-alpha and activation of Bid pathway. Apoptosis, 12, 1989-2001 (2007).

23) Koji T, Kondo S, Hishikawa Y, An S, Sato Y. In situ detection of methylated DNA by histo endonuclease-linked detection of methylated DNA sites: a new principle of analysis of DNA methylation. Histochem. Cell Biol., 130, 917-925 (2008).

24) Abo $T$, Nagayasu $T$, Hishikawa $Y$, Tagawa $T$, Nanashima A, Yamayoshi T, Matsumoto K, An S, Koji T. Expression of keratinocyte growth factor and its receptor in rat tracheal cartilage: possible involvement in wound healing of the damaged cartilage. Acta Histochem. Cytochem., 43, 89-98 (2010).

25) Shirendeb U, Hishikawa $Y$, Moriyama S, Win N, Thu MM, Mar KS, Khatanbaatar G, Masuzaki H, Koji T. Human papillomavirus infection and its possible correlation with $\mathrm{p} 63$ expression in cervical cancer in Japan, Mongolia, and Myanmar. Acta Histochem. Cytochem., 42, 181-190 (2009).

26) Furusu A, Miyazaki M, Abe K, Tsukasaki S, Shioshita K, Sasaki O, Miyazaki K, Ozono Y, Koji T, Harada T, Sakai H, Kohno S. Expression of endothelial and inducible nitric oxide synthase in human glomerulonephritis. Kidney Int., 53, 1760-1768 (1998).
27) Yokoyama H, Wada T, Furuichi K. Chemokines in renal fibrosis. Contrib. Nephrol., 139, 66-89 (2003).

28) Sean Eardley K, Cockwell P. Macrophages and progressive tubulointerstitial disease. Kidney Int., 68, 437-455 (2005).

29) Lu H, Kanazawa M, Ishida A, Tufescu A, Sasaki Y, Ito O, Kurosawa H, Sato T, Ootaka T, Kohzuki M. Combination of chronic exercise and antihypertensive therapy enhances renoprotective effects in rats with renal ablation. Am. J. Hypertens., 22, 1101-1106 (2009).

30) Rodríguez-Iturbe B, Pons H, Herrera-Acosta J, Johnson RJ. Role of immunocompetent cells in nonimmune renal diseases. Kidney Int., 59, 1626-1640 (2001).

31) Naya M, Tsukamoto $T$, Morita $K$, Katoh C, Furumoto T, Fujii S, Tamaki N, Tsutsui H. Olmesartan, but not amlodipine, improves endothelium-dependent coronary dilation in hypertensive patients. J. Am. Coll. Cardiol., 50, 1144-1149 (2007).

32) Fan YY, Kohno M, Nakano D, Hitomi H, Nagai Y, Fujisawa $Y$, Lu XM, Fu H, Du J, Ohmori K, Hosomi N, Kimura S, Kiyomoto $\mathrm{H}$, Nishiyama A. Inhibitory effects of a dihydropyridine calcium channel blocker on renal injury in aldosterone-infused rats. J. Hypertens., 27, 1855-1862 (2009).

33) Izuhara $Y$, Nangaku M, Inagi R, Tominaga N, Aizawa T, Kurokawa K, van Ypersele de Strihou C, Miyata T. Renoprotective properties of angiotensin receptor blockers beyond blood pressure lowering. $J$. Am. Soc. Nephrol., 16, 3631-3641 (2005).

34) Kadowaki D, Anraku M, Tasaki Y, Taguchi K, Shimoishi K, Seo H, Hirata S, Maruyama T, Otagiri M. Evaluation for antioxidant and renoprotective activity of olmesartan using nephrectomy rats. Biol. Pharm. Bull., 32, 2041-2045 (2009).

35) Konno S, Hirooka Y, Araki S, Koga Y, Kishi T, Sunagawa K. Azelnidipine decreases sympathetic nerve activity via antioxidant effect in the rostral ventrolateral medulla of stroke-prone spontaneously hypertensive rats. J. Cardiovasc. Pharmacol., 52, 555-560 (2008).

36) Manabe S, Okura T, Fukuoka T, Higaki J. Antioxidative effects of azelnidipine on mesangial cell proliferation induced by highly concentrated insulin. Eur. J. Pharmacol., 567, 252-257 (2007).

37) Matsui $T$, Yamagishi $S$, Nakamura K, Kikuchi S, Inoue H. Azelnidipine, a dihydropyridine-based calcium antagonist, inhibits angiotensin II-induced oxidative stress generation and downregulation of pigment epithelium-derived factor mRNA levels in microvascular endothelial cells. Drugs Exp. Clin. Res., 31, 215-219 (2005).

38) Miyata $T$, van Ypersele de Strihou $C$, Ueda $Y$, Ichimori $K$, Inagi R, Onogi H, Ishikawa N, Nangaku M, Kurokawa K. Angiotensin II receptor antagonists and angiotensin-converting enzyme inhibitors lower in vitro the formation of advanced glycation end products: biochemical mechanisms. J. Am. Soc. Nephrol., 13, 2478-2487 (2002).

39) Naito $Y$, Shimozawa M, Manabe H, Nakabe N, Katada K, Kokura S, Yoshida N, Ichikawa H, Kon T, Yoshikawa T. Azelnidipine, a new calcium channel blocker, inhibits endothelial inflammatory response by reducing intracellular levels of reactive oxygen species. Eur. J. Pharmacol., 546, 11-18 (2006).

40) Tanifuji C, Suzuki Y, Geot WM, Horikoshi S, Takahashi H, Tomino Y. Beneficial effects of combination therapy with olmesartan and azelnidipine in murine polycystic kidneys. Kidney Blood Press. Res., 32, 239-249 (2009).

41) Umemoto $S$, Tanaka $M$, Kawahara $S$, Kubo M, Umeji K, Hashimoto R, Matsuzaki M. Calcium antagonist reduces oxidative stress by upregulating $\mathrm{Cu} / \mathrm{Zn}$ superoxide dismutase in stroke-prone spontaneously hypertensive rats. Hypertens. Res., 27, 877-885 (2004).

42) Fujimoto S, Satoh M, Horike H, Hatta H, Haruna Y, Kobayashi S, Namikoshi T, Arakawa S, Tomita N, Kashihara N. Olmesartan ameliorates progressive glomerular injury in subtotal nephrectomized rats through suppression of superoxide production. Hypertens. Res., 31, 305-313 (2008). 